\title{
Is there a gap in the QCD Dirac spectrum above $\mathbf{T}_{c}$ ?
}

\author{
Tamas G. Kovacs*† \\ Institute for Nuclear Research, Debrecen \\ E-mail: kgteatomki.mta.hu

\section{Ferenc Pittler} \\ Institute for Nuclear Research, Debrecen \\ E-mail: pittler@atomki.mta.hu
}

\begin{abstract}
We study the previously found transition in the QCD Dirac spectrum from localized to delocalized modes. We use $N_{f}=2+1$ flavor simulations with physical quark masses to look at how the transition point in the spectrum (mobility edge) depends on the temperature and how it scales in the continuum limit. We find that in the continuum limit all eigenmodes below a finite physical scale in the spectrum are localized to the length scale of the inverse temperature. Due to their localized nature these eigenmodes cannot contribute to long distance spatial correlators. For long distance quark propagation the system effectively has a spectral gap equal to the mobility edge. We show that in the temperature range $1.5 T_{c}<T \leq 5 T_{c}$ the gap depends almost linearly on the temperature and extrapolates to zero at $T_{c}$. The gap is two orders of magnitude larger than the light quark mass. This could provide an explanation for the temperature dependence of hadronic screening masses above $T_{c}$
\end{abstract}

The 30th International Symposium on Lattice Field Theory

June 24 - 29, 2012

Cairns, Australia

*Speaker.

$\dagger$ TGK is supported by the Hungarian Academy of Sciences under "Lendület" grant No. LP2011-011. Both authors acknowledge partial support by the EU Grant (FP7/2007-2013)/ERC No. 208740. We also thank the BudapestWuppertal group for allowing us to use their code to generate the gauge configurations. 


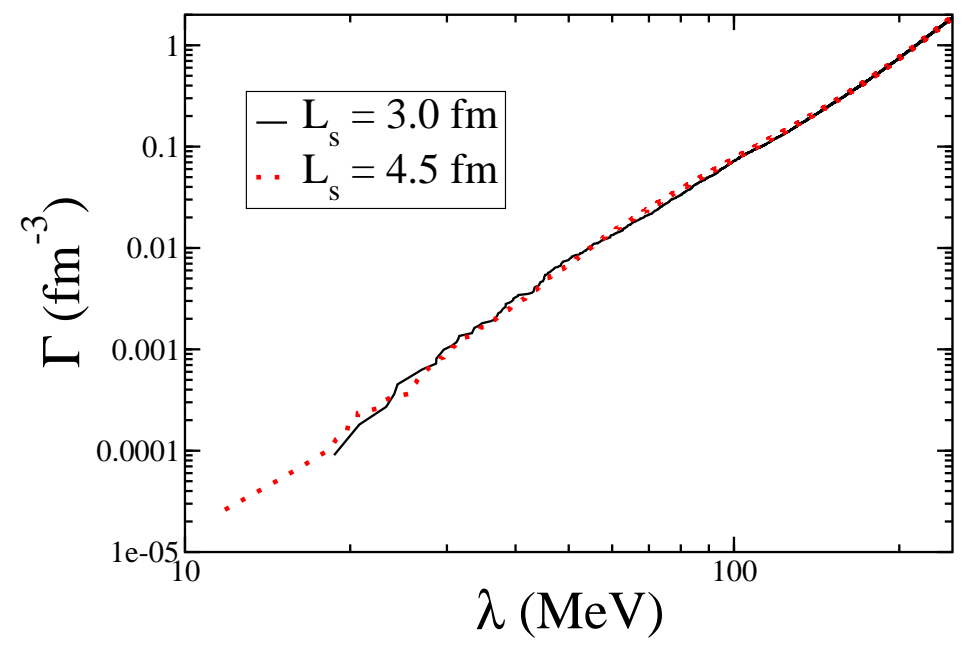

Figure 1: The integrated spectral density of the QCD Dirac operator computed at a temperature of $T=1.7 T_{c}$.

\section{Introduction}

Recently there has been a renewed interest in the spectrum of the QCD Dirac operator at and above the finite temperature transition [1]-[3]. The spectrum can certainly provide valuable information on the behaviour of the system. Most notably, the spectral density around zero is an order parameter for the spontaneous breaking of chiral symmetry. Below the transition where chiral symmetry is spontaneously broken, it is finite and above the transition it vanishes. However, the vanishing of the density at zero does not necessarily imply a gap in the spectrum there. In fact, there is no clear evidence of such a gap even well above the cross-over temperature, $T_{c}$. To demonstrate this, in Figure 1 we plot the integrated spectral density (normalized by the three-volume) of the Dirac operator at $T=1.7 T_{c}$, well above the transition. To resolve how the spectral density vanishes at zero we use log scale on both axes. The data is consistent with a vanishing gap and a spectral density going to zero as a power. A comparison of the data for two different spatial volumes indicates that even larger volumes might be needed to resolve the spectral density close to zero.

In the present paper we report on a detailed study of the lowest part of the Dirac spectrum in QCD above the critical temperature. Besides the spectral density we also look at correlations in the spectrum and localization properties of the Dirac eigenvectors. Our work is motivated by our recent findings that both in SU(2) Yang-Mills theory [4] and QCD [5] the lowest part of the spectrum consists of localized eigenmodes and the corresponding eigenvalues obey simple Poisson statistics. Here we would like to better understand the nature of this transition and its physical implications.

\section{Simulation details}

The present work is based on lattice simulations of QCD with $2+1$ flavours of dynamical quarks at the physical point set by the pion and the kaon mass. The lattice spacing was fixed using 
the kaon decay constant. We use the Symanzik improved gauge action and the two stout smeared staggered fermion action of the Budapest-Wuppeartal group with the parameters determined by them. Our simulations were performed at three values of the lattice spacing, $0.125 \mathrm{fm}, 0.082 \mathrm{fm}$ and $0.062 \mathrm{fm}$, on lattices of several different spatial size ranging from $2 \mathrm{fm}$ to $6 \mathrm{fm}$. To study the temperature dependence of the transition from localized to delocalized modes we considered the temperature range between $T=1.7 T_{c}$ and $5 T_{c}$. For more technical details we refer the reader to Ref. [7].

\section{Spectral statistics}

The main purpose of the present work is to study the details of the transition in the Dirac spectrum from the lowest, localized part to the delocalized regime higher up in the spectrum. The spectral statistics providing the clearest signal of such a transition is the distribution of the unfolded nearest neighbour level spacings defined as

$$
s=\frac{\lambda_{n+1}-\lambda_{n}}{\left\langle\lambda_{n+1}-\lambda_{n}\right\rangle} .
$$

For localized eigenmodes the distribution is known to be an exponential of the form

$$
P_{\mathrm{p}}(s)=\exp (-s)
$$

corresponding to eigenvalues distributed according to the Poisson distribution. In contrast, for delocalized eigenmodes it is given to a very good approximation by the Wigner surmise corresponding to the given Wigner-Dyson random matrix universality class. Lattice staggered fermions in the fundamental representation of the SU(3) gauge group are known to belong to the chiral unitary ensemble [8] and the Wigner surmise for that is

$$
P_{\mathrm{ws}}(s)=\frac{32}{\pi^{2}} s^{2} \cdot \exp \left(-\frac{4}{\pi} s^{2}\right)
$$

In any finite volume the transition from the exponential to the Wigner surmise distribution occurs gradually. To characterize the transition in a quantitative way, in Fig. 2 we plot how the variance of the unfolded level spacing distribution changes as we go up in the spectrum. The data shown corresponds to a temperature of $T=2.6 T_{c}$ and three different lattice spatial volumes ranging from $8 \mathrm{fm}^{3}$ to $64 \mathrm{fm}^{3}$. We also indicated with dashed lines the analytically computed values corresponding to the exponential distribution (localized case in the left of the plot) and the Wigner surmise (delocalized case in the right of the plot). The data smoothly interpolates between the two extremes confirming that indeed a delocalization transition occurs as on goes up in the spectrum. In Fig. 3 we show a blow-up of the transition region along with simple three-parameter fits to the data sets on the three spatial volumes. Inspecting the fit parameters (not shown here) also confirms what is already obvious by looking at the Figure; the transition gets stronger as the volume is increased. Its behaviour is consistent with the slope of the variance diverging at the inflection point in the thermodynamic limit. 


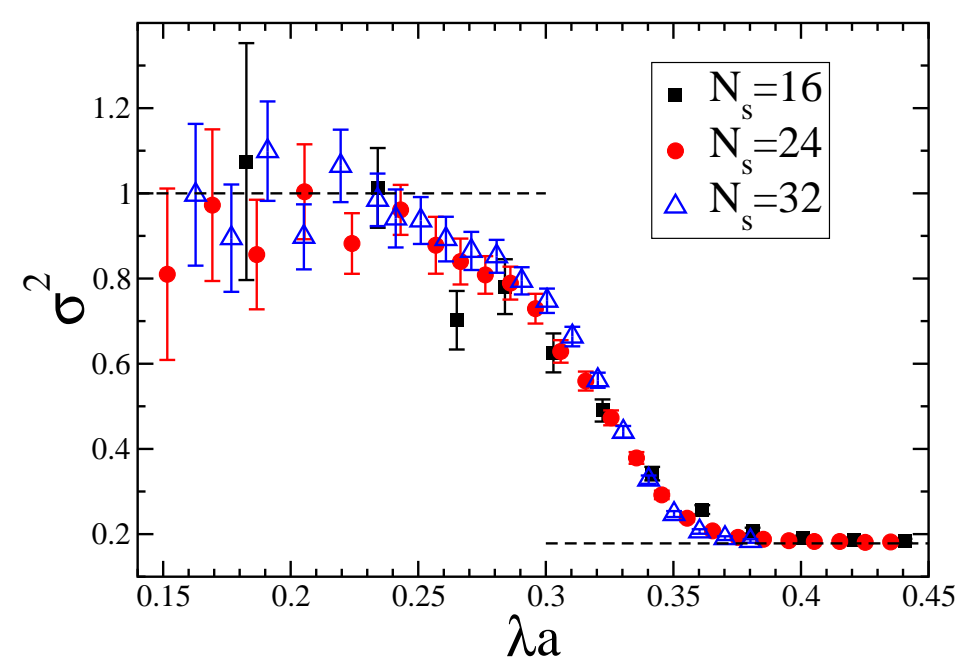

Figure 2: The variance of the unfolded level spacing distribution as a function of the location in the Dirac spectrum. The temperature was set to $T=2.6 T_{c}$ and the different symbols correspond to three spatial lattice sizes ranging from 2-4 fm across. The two dashed horizontal lines indicate the analytic results for the localized (exponential, left) and delocalized (Wigner surmise, right) case.

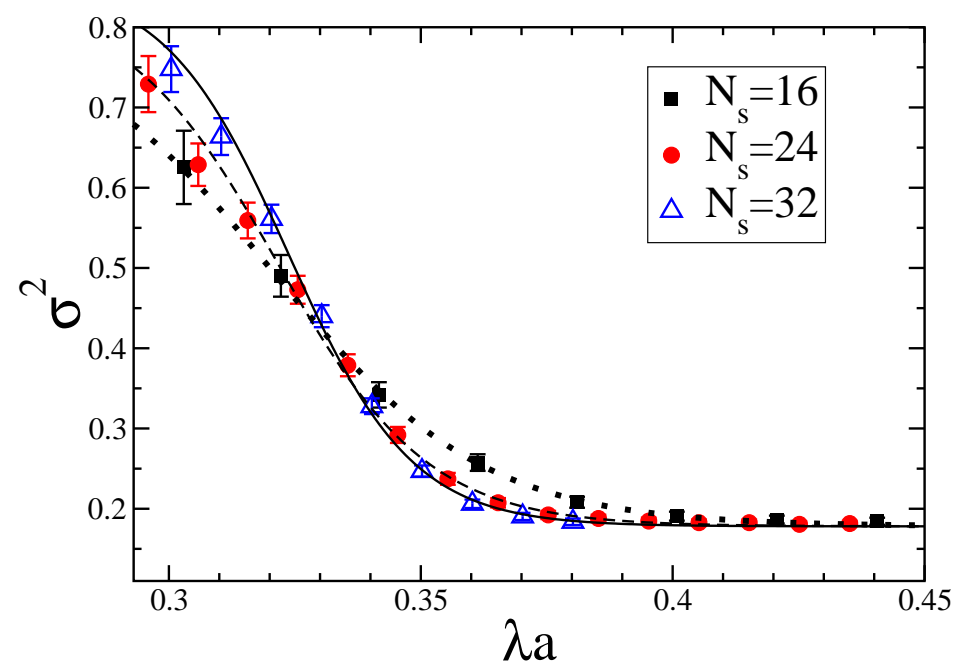

Figure 3: A blow-up of the transition region of Fig. 2. The continuous, the dashed and the dotted lines are separate fits for the three data sets.

\section{Possible physical impact of localization}

The lowest part of the Dirac spectrum is particularly important since hadronic correlators are built out of quark propagators. In the spectral decomposition of the propagator the lowest eigenmodes get a large contribution especially if the quark mass is small as is the case for the $u$ and the $d$ quark in QCD. If, however, the lowest quark modes are localized to a scale $d$ they cannot propagate quarks to distances $L \gg d$ and they effectively do not contribute to hadron correlators above that length scale. Therefore the physically most important questions about localization are 


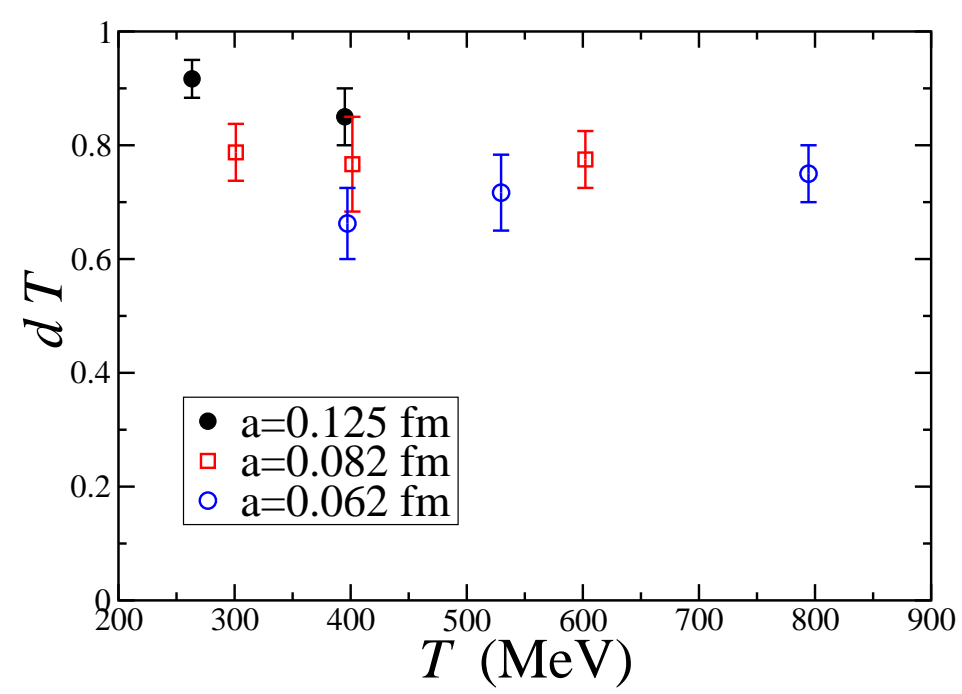

Figure 4: The localization length of the localized eigenmodes at the low-end of the Dirac spectrum as a function of the temperature. The different symbols correspond to data coming from simulations with different values of the lattice spacing.

the following;

1. What is the typical length scale $d$ where the lowest eigenmodes are localized (localization length)?

2. What is the point in the spectrum up to which eigenmodes are localized $\left(\lambda_{c}\right)$ ? In the literature on Anderson localization that is called the mobility edge.

These questions have to be answered in the continuum limit. One would also be interested in how the quantities $d$ and $\lambda_{c}$ depend on the physical temperature.

\section{Localization length}

The simplest way to measure the localization length of an eigenvector is based on the inverse participation ratio,

$$
I P R=\sum_{x}|\psi(x)|^{4},
$$

where $\psi(x)$ is a normalized eigenvector and the summation is over the whole space-time lattice. Since the IPR of a mode spreading homogeneously in a subvolume $v$ and being zero everywhere else is $1 / v$ we can define the physical localization length by

$$
d=a(I P R)^{-4}
$$

where $a$ is the lattice spacing. The quantity $d$ certainly depends on the spatial structure of the given eigenmode but it gives a rough idea of the length scale on which it spreads out. Therefore we will refer to $d$ as the localization length. In Fig. 4 we plot the localization length as a function of the temperature. Since the most natural unit to measure the localization length is the size of the box in 


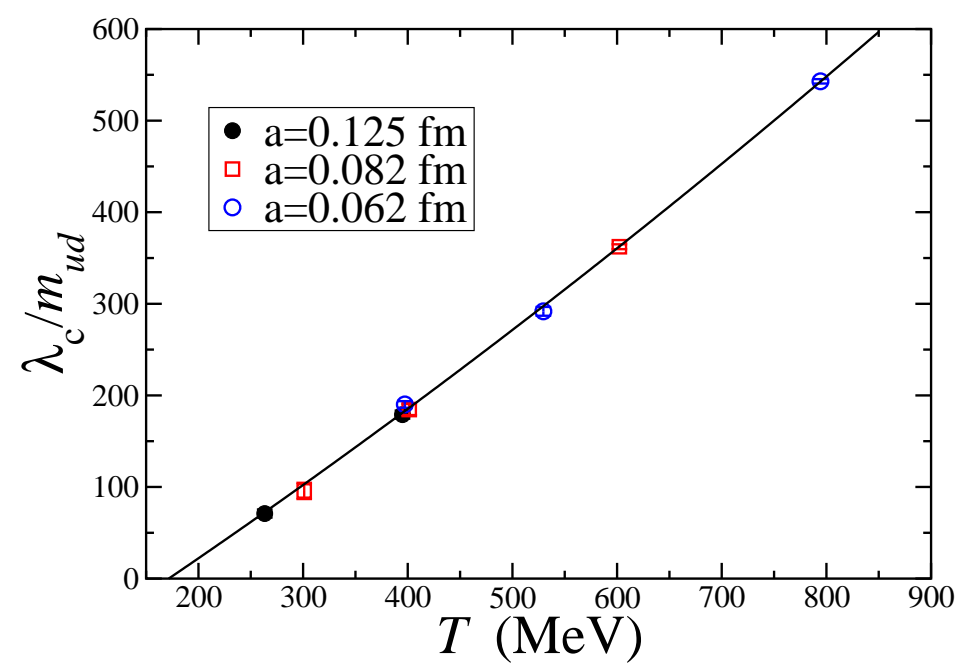

Figure 5: The ratio of the mobility edge and the light quark mass as a function of the temperature. Different symbols represent data taken at different values of the lattice spacing. The continuous curve is a second order polynomial fit to all the data. It crosses the horizontal axis at $T=172 \mathrm{Mev}$.

the temporal direction, i.e. the inverse temperature, we use this unit in the Figure. By comparing data obtained at different temperatures, it is apparent that the localization scale is indeed set by the inverse temperature. There might be a slight dependence of $d$ on the lattice spacing but it definitely does not increase in the continuum limit and it certainly always stays below the temporal box size.

\section{Continuum limit of the mobility edge}

We have seen that the lowest Dirac eigenmodes are localized to the length scale of the inverse temperature therefore they cannot propagate quarks to larger distances. The physical importance of this effect depends on how far up in the spectrum these localized modes extend. The quantity that characterizes this is the mobility edge, $\lambda_{c}$. In this respect the mobility edge plays a role similar to the quark mass, it acts effectively as a gap as far as quark modes propagating to long distances are concerned. $\lambda_{c}$ also renormalizes similarly to the quark mass (see [7] for details) and the ratio $\lambda_{c} / m_{\text {ud }}$ is a well-defined quantity in the continuum limit that can be used to characterize this effective gap.

In Fig. 5 we plot this ratio as a function of the temperature. We determined $\lambda_{c}$ using the fits in Fig. 3 and identifying the inflection point of the fitted functions. This turned out to be volumeindependent to a very good precision. The data obtained at three different values of the lattice spacing fall on the same smooth curve showing that discretization effects are well in control. The temperature-dependence of the mobility edge turns out to be almost linear but a small quadratic term is needed to render the $\chi^{2}$ of a global fit to all the data in the Figure acceptable. Extrapolating the fitted curve to smaller temperatures beyond the range of the data shows that the mobility edge vanishes at a temperature of $172 \mathrm{MeV}$ which is consistent with the location of the finite temperature cross-over $[9,10]$. Below that the lowest Dirac eigenmodes are already expected to be delocalized and their spectral statistics is described by Wigner-Dyson random matrix statistics. 


\section{Conclusions}

We showed that the lowest part of the QCD Dirac spectrum consists of localized eigenmodes and the spectral statistics of the corresponding eigenvalues obeys Poisson statistics. There is a mobility edge, $\lambda_{c}$ separating these localized modes from extended modes higher up in the spectrum. The transition at $\lambda_{c}$ appears to be similar to a phase transition as it becomes stronger if the spatial volume is increased. In the localized regime the localization length is at or below the inverse temperature and modes below $\lambda_{c}$ cannot propagate quarks to distances larger than $1 / T$. In this respect the mobility edge is effectively a gap in the spectrum at least as far as long-distance physics is concerned. It plays a role similar to the quark mass but it is two orders of magnitude larger than that, already at moderately high temperatures $\left(2-5 T_{c}\right)$. The scaling of the mobility edge and the localization length indicates that localization might provide a microscopic explanation of the observed increase of hadronic screening masses with the temperature [11].

\section{References}

[1] S. Aoki, H. Fukaya and Y. Taniguchi, arXiv:1209.2061 [hep-lat].

[2] H. Ohno, U. M. Heller, F. Karsch and S. Mukherjee, PoS LATTICE 2011 (2011) 210 [arXiv:1111.1939 [hep-lat]].

[3] Z. Lin [HotQCD Collaboration], PoS LATTICE 2011 (2011) 204 [arXiv:1111.0988 [hep-lat]].

[4] T. G. Kovacs and F. Pittler, Phys. Rev. Lett. 105 (2010) 192001 [arXiv:1006.1205 [hep-lat]].

[5] T. G. Kovacs and F. Pittler, PoS LATTICE 2011 (2011) 213 [arXiv:1111.3524 [hep-lat]].

[6] S. Borsanyi, G. Endrodi, Z. Fodor, A. Jakovac, S. D. Katz, S. Krieg, C. Ratti and K. K. Szabo, JHEP 1011, 077 (2010) [arXiv:1007.2580 [hep-lat]].

[7] T. G. Kovacs and F. Pittler, arXiv:1208.3475 [hep-lat].

[8] J. J. M. Verbaarschot and T. Wettig, Ann. Rev. Nucl. Part. Sci. 50 (2000) 343 [hep-ph/0003017].

[9] S. Borsanyi et al. [Wuppertal-Budapest Collaboration], JHEP 1009, 073 (2010) [arXiv:1005.3508 [hep-lat]].

[10] A. Bazavov, T. Bhattacharya, M. Cheng, C. DeTar, H. T. Ding, S. Gottlieb, R. Gupta and P. Hegde et al., Phys. Rev. D 85, 054503 (2012) [arXiv:1111.1710 [hep-lat]].

[11] M. Cheng, S. Datta, A. Francis, J. van der Heide, C. Jung, O. Kaczmarek, F. Karsch and E. Laermann et al., Eur. Phys. J. C 71, 1564 (2011) [arXiv:1010.1216 [hep-lat]]. 